\section{ON THE MODE OF PROPAGATION OF NERVOUS IMPULSES.*}

By J. J. CHARLES, M.A., M.D., Professor of Anatomy and Physiology, Queen's College, Cork.

Different hypotheses have been propounded at different times to explain the mode of propagation of nervous impulses. Those which meet with most acceptance at the present day are two in number. First, the vibratory hypothesis, as it may be called, according to which the impulse depends on the displacement or vibration of the nerve particles; second, the chemical hypothesis, by which the impulse is represented as the result of a series of chemical changes in the nerve particles. Professor Huxlcy holds the first view, and in an article "On Sensation and Sensiferous Organs", which appeared in the April number of the Nineteenth Century, he quotes a passage from Hartley's Observations on Man as containing a concise exposition of this view. The passage is as follows: "External objects impressed upon the senses occasion, first on the nerves on which they are impressed, and then on the brain, vibrations of the small, and, as we may say, infinitesimal medullary particles. These vibrations are motion backwards and forwards of the small particles; of the same kind with the oscillations of pendulums and the tremblings of the particles of sounding bodies. They must be conceived to be exceeding short and small, so as not to have the least efficacy to disturb or move the whole bodies of the nerve or brain." Further on, in this article, Professor Huxley says : "It is quite possible that the pariicular mode of motion of the object is reproduced in the sensorium, exactly as the diaphragm of the telephone reproduces the mode of motion taken up at its receiving end."-This hypothesis, it must be admitted, is at first sight plausible, and without doubt serves to explain several phenomena observed during the active condition of the nervous system. But, notwithstanding the support it has received, I am disposed to believe that the weight of evidence is decidedly in favour of the other-the chemical hypothesis; and I shall take the liberty of directing attention to a number of facts, which, when considered with reference to the cumulative evidence they afford, go far, in my opinion, to disprove the vibratory, and at the same time confirm the chemical hypothesis. What is meant by the latter hypothesis I shall forthwith proceed to explain, and shall subsequently state the facts which make for and against the respective hypotheses.

By the chemical hypothesis, it is assumed that the stimulus, whether mechanical, thermal, or electrical, when applied to a nerve, excites a chemical change in its molecules or elementary particles, and that this change travels along the nerve like fire along a piece of wood or a train of gunpowder. The nervous impulse or force is supposed to be produced by the chemical reaction between the nerve particles and the blood, just as heat is generated by the chemical reaction between the particles of wood and the oxygen of the atmospheric air; and the nervous impulse may be considered an expression of the chemical change in the nerve, somewhat in the same manner as heat is an expression of the chemical change in the case of the wood or gunpowder. But let us see to what extent this hypothesis is supported by facts.

1. A slight elevation of the temperature of the nerve occurs during the conduction of an artificial stimulus. Schift and Oehl, + by means of the thermo-electric apparatus, noticed this increase of temperature in large nerves like the sciatic.§ Now, it is difficult to account for a rise of temperature that could be detected on the vibratory hypothesis, or on the supposition that nerves are nothing more than mere conductors, like the wires of the electric telegraph.

2. Chemical changes take place in nerves when in a state of activity; these are probably of the same nature as those that have been discovered in muscle, but are much less in amount. It can be readily understood that it is very difficult to make satisfactory analyses of the nerves on account of their small size-hence, no doubt, the discordant results arrived at by different chemists. But the white substance of the brain,

* Read in the Section of Physiology at the Annual Meeting of the British Medical Association in Cork, August 1879 .

$\dagger$ Archives de Plyysiologic, vol. iii, p. 75. Paris: r87o.

\$ Quoted in Carpenter's Physiology, p. 612. 1876.

\$ Helmholtz and Heidenhaim, however, it is but just to nention, were unable to detect it. which is composed essentially of nervous filaments, has been carefully analysed, and has yielded tangible results. Ranke showed that if the brains of pigeons were deprived of blood, they absorbed oxygen and gave out carbonic acid. Nerves, after prolonged action, are acid in reaction (the acid is lactic according to Ranke); whereas nerves in a state of repose possess a neutral reaction. Urea and cholesterin also are said to appear in nerves after action. On the other hand, it must be acknowledged that some chemists have not found the chemical changes I have indicated; and Dr. M. Foster, adopting their view, states that the chemical changes in nerve are insignificant, or have not been satisfactorily made out.* Now, on the vibratory hypothesis, it may be urged that these changes, comparatively small in amount according to some chemists, result from the waste of the nervous tissue consequent on the vibration of the nerve particles. But it is scarcely possible that the mere vibration of particles, either in nerve-cells or in the nerves themselves, should give rise to so much waste as is indicated by the chemical products formed; or that the brain should receive nearly one-sixth, by weight, of the entire blood of the body, and should cease to perform its functions the moment its vascular supply is cut off, if, as on this hypothesis, blood were only required by it to supply the deficiency created by the "wear and tear". Even Foster appears to take this view; for he says "the actual amount of energy developed by a most powerful nervous impulse is exceedingly slight, and hence chemical changes insignificant in amount may be the cause of all the phenomena and yet remain too slight to be readily recognised". + Moreover, we can scarcely imagine that the potential part of a nerve should be constituted by so highly vitalised a product as protoplasm, which cxhibits so great a rcadiness to undergo chemical change, if its sole function be merely to serve a mechanical purpose, such as the transmission of vibrations ; for any other tissue might have been as suitably employed for such a purpose, since it has been shown that nerves are but indifferent conductors of electricity, and furthermore have not a greater conducting power than other moist animal textures. $\neq$ Besides the fact that the chemical constituents of nerves and nerve-cells are qualitatively almost the same, renders it more than probable that the nerves play some part in the generation of impulses.

3. The phenomena of electrotonus appear to favour the vibratory hypothesis. Cyon's law to explain the anelectrotonic and cathelectrotonic zones of Pflüger is hased, indeed, on the assumption that the nerve molecules may exist in three states ; first, a neutral state, their ordinary condition; second, a state of increased mobility (cathelectrotonus); and third, a state of diminished mobility (anelectrotonus). But Matteucci, Hermann, Onimus, Radcliffe, and others have shown by experiments, which I nced not describe, that all the phenomena of electrotonus may be explained on purely physical principles by considering the nerves merely as conductors of electricity ; and that the changes in conductivity and excitability which occur in a nerve in this condition depend either on the electrolytic action of the electric current on the nerve [in which case acid would be formed at the positive pole, where it would lower the excitability, and alkali at the negative pole, where it would raise the excitability (Onimus)], or on "an internal polarisation which appears at the border between the sheath and the contents of the nervetube" (Hermann). $\S$

4. It has been ascertained that the excitability of a nerve when exhausted by a direct current can be restored more promptly by stimulation with the inverse current than by absolute repose, and vice versâ. In the second place, it has been demonstrated that any nerve when exhausted by a direct current is still sensitive to the inverse one. The explanation of these phenomena, in terms of the vibratory hypothesis, is that the molecules are assisted in returning to their original or ordinary position by the action of the inverse current ; but the supposition that the acid and alkali generated at the positive and negative poles, by the electrolytic action of the direct current, are neutralised respectively by the alkali and acid formed on a reversal of the current would seem to afford a more satisfactory explanation.

5. Marked changes in the chemical composition of the nerves destroy their excitability. Strong alkalies and acids, etc., abolish the excitability of a nerve, whilst dilute solution of chloride of sodium increases it. The chloride of sodium may possibly act by dissolving the albuminous product allied to myosin, which is formed in the nerve during its active condition, and so help to prepare it for undergoing chemical changes anew.

6. The excitability of nerves is increased by heat, and diminished by

Text-Book of Physiology, p. $4^{3} . \quad$ I873.

† Op. cit., p. 79 .

† Müller's Physiologry, translated by Baily, vol. i, p. 684. $\quad$ r840.

$\$$ II:tmax Physiology, translated by Gamgee, p. 346. $\quad$ 1878. 
cold. This is explicable on the chemical hypothesis, for heat promotes combustion; but herein is the vibratory hypothesis at fault, since heat diminishes, whilst cold increases the conductivity of metals.

7. Fatigue renders nerves less excitable, exhaustion being usually accompanied by a retardation of the impulse-wave. The excitability of nerves depends on the state of their nutrition; and this, again, on not mnly the quantity but the quality of the blood. These facts are more ea ily interpreted by the chemical than by the vibratory hypothesis.

8. All varieties of stimuli-mechanical, thermal, electrical-give rise to the same kind of nervous impulse. This fact alone renders it, on the one hand, highly improbable that the impulse is caused by the vibration of the par $i$ les, and on the other hand, probable that it originates in chemical action.

9. Chemical combination can take place with sufficient celerity to account for the rate with which a nervous impulse travels along a nerve. The velocity of propagation of chemical action depends to a considerable extent, as Bunsen showed in the case of gases, on whether the comloustible substances are pure or are mixed with less combustible bodies; for, by judiciously combining them in different proportions in a series of experiments, Bunsen was able to reduce by degrees the rate with which iynition was propagated until he could follow it with the naked eye. IIe further, by means of a niost ingenious method, determined the velocity with which the ignition of gases travelled.* He found that this velocity for pure hydrogen and oxygen was 34 metrcs (III feet) per second-a velocity, it should be remarked, identical with the average velocity of the nervous impulse in the motor and sensory nerves of man as determined by Helmholtz. The analogy between the two modes of propagation is certainly striking; but, of course, I do not intend to suggest that the chemical change in nerve is nothing more than an union of hydrogen and oxygen. I merely take it as an instance of the rapid propagation of chemical action; and I would call attention to the whole series of Bunsen's experiments as tending undoubtedly to throw considerable light on the manner in $w$ hich chemical action may take place in nerve.

I0. Lastly, a nervous impulse presents the essential character of increasing in intensity, in a motor nerve, in proportion to its progress along it. Thus, if a stimulus be applied to the nerve at the point $a$, and again at the point $h$, the contraction of the muscle will be much greater in the former case than in the latter; in fact, the maximum of

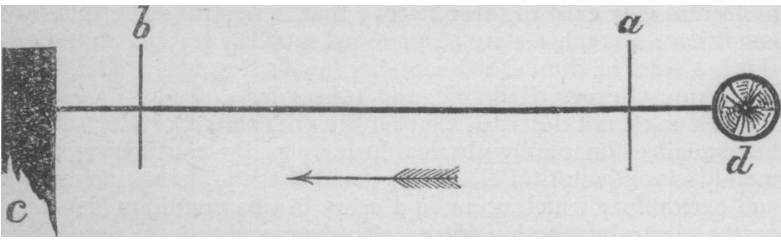

c. Muscle. d. Spinal cord.

contraction, as Pfliger pointed out, corresponds to the maximum of distance from the muscle.t These results are observed as well in a nerve in connection with the spinal cord as in one whose connection with it has been severed; they cannot, therefore, be due to the mere section of the nerve.

Heidenhaim, too, has shown that, in a nerve in connection with the spinal cord, "the amount of contraction of the muscle is at first large, then becomes smaller, and finally increases somewhat again, as the stimulation is carried from the roots of the nerves to the muscular periphery".t To explain this phenomenon on the vibratory hypothesis seems to be impossible; for, if a nerve were merely a conductor, like an iron wire, the impulse propagated along it should, owing to the resistance, which is of a frictional character, gradually diminish from its origin. Two views, however, have been advanced to account for this peculiar phenomenon. The first is, that a greater impulse is produced on-stimulation of the proximal than of the distal portions of a nerve because the former are the more excitable. The cause of this increased excitability is believed by some physiologists to be "the proximity of the central organ with which the nerve is connected"; $\$$ by others, that "the structure of the nerve, and especially of its sheath, may be more delicate in those parts which are more protected, and that it consequently there more readily responds to impressions". II But the results

* Prilosoprical.Magasine, vol. xxxiv, p. 492. 1867.

+ Marey, as the result of his experiments, arrived at a different conclusion from Pflüyer; but most yhysiologists are now convinced of the accuracy of 'pflüger's periments.

I Foster's Physio'ogy', r. 7r.

Hermann's Plysiology, p. 344. 1878 .

I Curpenter's Fhysiclog", I. 612. 1876 . in question do not follow stimulation of the proximal end only, so that the proximity of the central organ cannot be the true cause; and, though the structure of a nerve is most delicate at its origin and its termination, I think it has yet to be shown that nerves in their cotirse become gradually less delicate or their sheaths gradually thicker; yet this ought necessarily to be the case, else the cause assigned for the increased excitability cannot be the true one. Thus, in order that the latter explanation hold good, it would be requisite that the sheath of the nerve should, as shown in the diagram, increase in thickness from $a$ to $i:$ but if it do not, then, cateris paribus, a stimulus applied at $c$ should give a greater result than when applied at $b$.

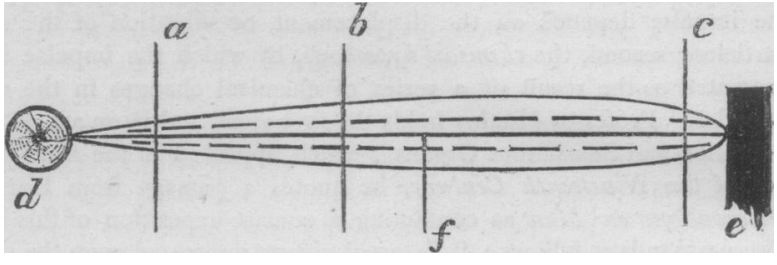

c. Muscle. d. Spinal cord.

The second view regarding this phenomenon was given by Pfüger : it assumes that the impulse gains force in its descent along the nerve like an avalanche. This, however, is rather an illustration of the phenomenon than an explanation of its cause. The only objection to it appears to be that the "negative variation" probably diminishes its intensity as it passes along the nerve ( $\mathrm{Du}$ Bois-Reymond); but the observations on this point have not been sufficiently numerous :0 entitle us to draw a general conclusion from them. It occurs to me that the explanation which is on the whole the most satisfactory is afforded by a consideration of the mode of propagation of the nervous impulse as assumed by the chemical hypothesis. We can imagine a nerrous impulse, generated as the result of chemical change in the particles or in the cross-section of a nerve at the seat of stimulation; and, as it passes towards the muscle, becoming slightly increased at each crosssection of the nerve, owing to chemical action, the total addition made to the original impulse being in some degree proportional to the distance it has travelled along the rerve. We might illustrate this hypothesis by a chain of Daniell's cells or a thermo-pile, in which the ceils and the junctions would each of them respectively correspond to a cross-section of a nerve undergoing chemical change; and as in this illustration the amount of electricity produced is, within certain limits, proportional to the number of cells or metallic combinations, so is the nervous impulse increased by an addition at each cross-section as it proceeds onwards.

Another illustration is presented by the burning of a piece of wood. The heat developed by the combustion of the first portion raises the temperature of the succeeding portions, so that they burn more rapidly -more vigorously; that is, they give out more energy when the fire reaches them.

Whether the nervous impulse is a force in some respects resembling electricity, generated by, but distinct from, chemical action, or whether it is merely altered chemical force, possessing no separate and independent existence, I do not pretend to say, though I am disposed to take the first view; but either conception may be held consistently with the chemical hypothesis of the propagation of nervous impulses.

Note.-Herbert Spencer, I may add, holds the chemical hypothesis in a somewhat modified form. He believes the chemical change in a nerve-cell to be of the nature of decomposition, and the chemical change in a nerve to be of the nature of isomeric transformation. The first change-that of decomposition-he says, is " usually accompanied by a great dissipation of motion, whereas the motion given out or taken up along with the other (the isomeric transformation) is relatively insignificant". (Principles of Psychology, vol. i, p. 21 ; 1870.) To this viewat least in so far as the motion of molecules is made to depend on chemical change-I do not think any serious objection can be raised; however, granting the existence of isomeric transformation as a factor in the production of nervous impulses, it seems highly probable, from what I stated above, that chemical decomposition takes place in nerves as well as in nerve-cells. Chemical change in nervous matter will no doubt give rise to more or less molecular or vibratory movement of the nerve-particles; accordingly, to a certain extent, the chemical hypothesis may be regarded as including the vibratcry hypothesis. But this is far from being eqvivalent to affirming that a nervous impulse is propagated solely by vibration of molecules irrespective of chemical change except as the result of waste of nervous tissue. 